\title{
Increased false positive Down syndrome screening in women with sickle cell anemia ${ }^{\dagger}$
}

\author{
Anna W. Kneitel ${ }^{1 *}$, Laila Rhee-Morris ${ }^{2}$, Roberta Obadia ${ }^{3}$ and Dena Towner ${ }^{4}$ \\ 'Department of Obstetrics and Gynecology, University of Michigan, Ann Arbor, MI, USA \\ 2Department of Obstetrics and Gynecology, University of California, Davis, Sacramento, CA, USA \\ ${ }^{3}$ Kaiser Permanente Woodland Hills, Los Angeles, CA, USA \\ ${ }^{4}$ Department of Obstetrics and Gynecology, University of Hawaii, Honolulu, HI, USA \\ *Correspondence to: Anna Weber Kneitel. E-mail: akneitel@gmail.com \\ †Poster presented at the 32nd Annual Meeting of the Society for Maternal-Fetal Medicine, Dallas, TX, 6-1 1 February 2012.
}

\begin{abstract}
Objectives This study seeks to determine whether there is a higher rate of false positive serum screening for Down syndrome in women with sickle cell anemia and, if so, which markers contribute to the false positive screen.
\end{abstract}

Methods This is a retrospective cohort study of women who had serum screening between 1998 and 2011. Subjects were women with sickle cell anemia $(n=13)$, and controls were African American women who did not have that disease $(n=91)$. The populations were compared using basic inferential statistics.

Results The positive screen rate was $38.5 \%(5 / 13)$ in women with sickle cell anemia and $7.7 \%(7 / 91)$ in the control population (odds ratio 7.5, 95\% confidence interval 1.6-35.8, $P=0.001$ ). At the average age of the cases (25 years), the expected false positive rate is only $2 \%$. The human chorionic gonadotrophin values were significantly higher in cases than controls ( 2.00 and $1.30 \mathrm{MoM}, P=0.017$ ), whereas levels of other serum analytes were similar. None of the screen positive results were associated with a fetus or neonate affected by Down syndrome.

Conclusions The false positive Down syndrome serum screen rate is significantly higher in patients with sickle cell anemia than in African American women without that disease. The human chorionic gonadotrophin values were significantly higher in cases than controls, suggesting that placental factors may contribute to the elevated false positive rate. (C) 2015 John Wiley \& Sons, Ltd.

Funding sources: None

Conflicts of interest: None declared

\section{INTRODUCTION}

Maternal serum screening is used to identify fetuses at risk for a variety of common disorders, the most frequent of which is Down syndrome. ${ }^{1,2}$ The California Prenatal Screening Program provides prenatal screening to all women residing in the state and has a false positive rate of $5 \%$ for Down syndrome. Maternal age is a significant factor in the risk calculation: women $<28$ years old have a $2 \%$ risk of screening positive whereas women over age 40 years have a screen positive rate of $>20 \%$.

After the advent of serum screening for birth defects, it became evident that pregnancies with abnormal serum analytes have increased risk of an underlying abnormal placentation and related clinical manifestations. Specifically, low pregnancy-associated plasma protein A (PAPP-A), and estriol, and elevated alpha-fetoprotein (AFP), human chorionic gonadotrophin (hCG), and inhibin A are associated with preeclampsia, fetal growth restriction, and preterm birth. ${ }^{3}$
This study was conceived when we noticed that women referred to the University of California, Davis, prenatal diagnosis program for sickle cell anemia had high rates of false positive prenatal screening results for Down syndrome. We hypothesize that these pregnancies may be at increased risk of placental insults because of sickle cell disease and may thus be at an increased risk for abnormal serum screening results.

Our study has two objectives: to determine whether women with sickle cell anemia have a higher rate of false positive prenatal screening for Down syndrome and, if so, to determine which serum markers contribute to these false positive screens. This information may be helpful in counseling women with sickle cell anemia as they decide what kind of prenatal screening to pursue.

\section{MATERIALS AND METHODS}

This is a retrospective cohort study that was approved by the institutional review board of the Office of Research at the 
University of California, Davis. Since 1994, the UC Davis Department of Obstetrics and Gynecology has maintained a database for quality assurance purposes of all women seen for prenatal genetic counseling and testing. The department maintains a separate database of all women seen in the high risk obstetrics clinic; all patients with sickle cell anemia in prenatal care at UC Davis would have been seen in this clinic. Thus, ascertainment of all cases with sickle cell disease is ensured by using both data sets. We used the portion of this dataset that includes women seen from 1998 to 2011 to identify cases and controls. We chose 1998 as the starting date, because that was the first year in which a patient with sickle cell anemia had serum screening. Each patient was used as a subject only once; if she had more than one pregnancy during the study period, we used the data from the earlier pregnancy. We excluded patients with multiple gestations.

Cases and controls were identified from these two data sets. Cases were comprised of those women with sickle cell anemia who had prenatal serum screening performed. All of these women were African American. The controls were all African American women without sickle cell disease who had serum screening and were seen in prenatal diagnosis or had care in the high risk obstetrics clinic. We identified these patients for the control group by searching our prenatal genetic counseling database and our high risk obstetrics clinic database for African American women and included those who had serum screening.

For each case and control, we collected information from the databases including age, Down syndrome risk, and the value of each serum analyte measured in multiples of the median (MoM). We collected values for second-trimester AFP, hCG, estriol, and inhibin A and, when available, first-trimester PAPP-A and free $\beta$-hCG. Whenever serum screen results were missing from the database, a review of the patient's institutional medical record was conducted. Serum screen results that could not be obtained through these avenues were requested directly from the California Prenatal Screening Program. Not all serum analyte values were available for all women. Patients with no Down syndrome risk documented were excluded.

A positive Down syndrome serum screen was defined by the cutoff used in the California Prenatal Screening Program. For second-trimester quad screening, this was 1 in 200. For all patients with a positive screen, a review of the mother's and infant's charts was conducted to determine whether the infant had Down syndrome. This allowed us to determine whether the screen was falsely or truly positive.

The false positive serum screen rate was then calculated for the case population and the control population, and the rates compared using a chi-squared test. Mean second-trimester serum analyte values were calculated for cases and controls and compared using a two-sample $t$-test assuming equal variances. Because only two of the cases had first-trimester screening, we did not calculate means for PAPP-A or first-trimester hCG.

\section{RESULTS}

Of the 13 women with sickle cell anemia, five (38.5\%) had a positive Down syndrome screen. Of those with a positive screen, four had normal karyotype by amniocentesis. One woman declined amniocentesis, and chart review revealed that her infant had no clinical manifestations of Down syndrome (Table 1).

We identified 91 women for our control population. Of these, seven $(7.7 \%)$ had a positive Down syndrome screen. Of those with a positive screen, five could be confirmed as definite false positives, two because of normal karyotype and three by chart review (Table 2). The other two were assumed to be false positives as both had a Down syndrome risk $\leq 1 \%$. Maximizing

Table 1 Cases: patients with sickle cell anemia

\begin{tabular}{|c|c|c|c|c|c|c|c|c|}
\hline & \multirow[b]{2}{*}{ Age } & \multirow[b]{2}{*}{ T21 risk } & \multicolumn{4}{|c|}{ Serum analytes (MoM) } & \multirow[b]{2}{*}{ GA at delivery } & \multirow[b]{2}{*}{ Notes } \\
\hline & & & AFP & hCG & uE3 & Inhibin & & \\
\hline Case 1 & 23 & $1: 400$ & 1.04 & 0.96 & 0.61 & 0.87 & 39 weeks 2 days & \\
\hline Case 2 & 20 & $1: 10000$ & 0.88 & 0.89 & 1.55 & & 38 weeks 5 days & \\
\hline Case 3 & 19 & $1: 770$ & 0.68 & 0.97 & 0.67 & & Unknown & \\
\hline Case 4 & 20 & $1: 1300$ & 0.70 & 1.40 & 0.99 & & 37 weeks 2 days & IUGR \\
\hline Case 5 & 20 & $1: 100000$ & 1.11 & 0.53 & 0.91 & 0.87 & 37 weeks 0 days & \\
\hline Case 6 & 20 & $1: 140$ & 0.49 & 2.39 & 0.85 & 0.93 & 38 weeks 6 days & $\begin{array}{l}\text { No karyotype performed; } \\
\text { Infant without T21 }\end{array}$ \\
\hline Case 7 & 34 & $1: 870$ & 0.86 & 1.11 & 0.93 & & Unknown & \\
\hline Case 8 & 24 & $1: 10$ & 0.48 & 6.39 & 0.36 & & 39 weeks 4 days & $46, \mathrm{XX}$ by amniocentesis \\
\hline Case 9 & 30 & $1: 60$ & 1.2 & 3.97 & 0.97 & 4.97 & 20 weeks 2 days & $\begin{array}{l}\text { Pregnancy terminated for social reasons; } \\
\text { karyotype from outside institution } \\
\text { reportedly normal }\end{array}$ \\
\hline Case 10 & 35 & $1: 1300$ & .91 & 1.59 & 1.92 & 0.89 & 40 weeks 0 days & \\
\hline Case 11 & 32 & $1: 33$ & 1.99 & 3.54 & 0.28 & & 15 weeks 1 day & IUFD; $46, X X$ by amniocentesis \\
\hline Case 12 & 28 & $1: 19$ & 1.84 & 1.86 & 0.30 & 2.93 & 20 weeks 0 days & IUFD; $46, X Y$ by amniocentesis \\
\hline Case 13 & 19 & $1: 10000$ & 0.84 & 0.35 & 1.89 & & 36 weeks 2 days & Severe preeclampsia \\
\hline
\end{tabular}

AFP, elevated alpha-fetoprotein; hCG, human chorionic gonadotrophin; GA, gestational age; IUGR, intrauterine growth restriction; IUFD, intrauterine fetal demise. 
Table 2 Control patients with positive serum screen

\begin{tabular}{|c|c|c|c|c|c|c|c|c|}
\hline & \multirow[b]{2}{*}{ Age } & \multirow[b]{2}{*}{ T21 risk } & \multicolumn{4}{|c|}{ Serum analytes (MoM) } & \multirow[b]{2}{*}{ GA at Delivery } & \multirow[b]{2}{*}{ Notes } \\
\hline & & & AFP & hCG & uE3 & Inhibin & & \\
\hline Patient 1 & 23 & $1: 38$ & 1.77 & 2.82 & 0.39 & 1.18 & 27 weeks 4 days & Infant without T21 \\
\hline Patient 2 & 36 & $1: 31$ & 0.89 & 2.91 & 0.73 & 0.33 & 38 weeks 1 day & Infant without T21 \\
\hline Patient 3 & 38 & $1: 100$ & 1.59 & 1.73 & 0.61 & & 39 weeks 2 days & Infant without T21 \\
\hline Patient 4 & 40 & $1: 24$ & 0.83 & 1.64 & 0.66 & & 39 weeks 1 day & $46, \mathrm{XX}$ by amniocentesis \\
\hline Patient 5 & 42 & $1: 20$ & 1.13 & 1.24 & 0.39 & & 36 weeks 5 days & $46, X Y$ by amniocentesis \\
\hline Patient 6 & 31 & $1: 100$ & 0.84 & 0.67 & 0.40 & & Unknown & \\
\hline Patient 7 & 40 & $1: 150$ & 0.76 & 1.01 & 0.97 & & Unknown & \\
\hline
\end{tabular}

AFP, elevated alpha-fetoprotein; hCG, human chorionic gonadotrophin; GA, gestational age.

the false positive rate in this way makes it less likely that the false positive rate for the control group would be underestimated, and less likely that the significant difference between rates in cases and controls would be overstated.

The rate of false positive screens was significantly higher in women with sickle cell anemia than controls: odds ratio 7.5, 95\% confidence interval 1.6-35.8, $P=0.001$ (Table 2). For women age 18-28years in California, the expected screen positive rate is $2 \%$, thus both cases and controls had a higher than expected screen positive rate.

Table 3 displays the characteristics of the two groups.

Among serum analytes, hCG levels were significantly higher in women with sickle cell anemia than in controls. There was also a trend toward higher inhibin in cases than in controls. AFP and estriol values were similar in the two populations.

Among the 13 cases, there were seven deliveries at greater than 37 weeks gestational age and one preterm delivery at 36 weeks 2 days gestational age. There were two intrauterine fetal demises, at 17 and 20 weeks, both with normal karyotypes. One patient electively terminated her pregnancy at 20 weeks gestation for reasons unrelated to the fetal Down syndrome risk; she did have an amniocentesis at an outside institution, but the result is unknown. Two of the pregnancy outcomes are unknown. The five cases with false positive screens included two women with term deliveries, the terminated pregnancy, and the two fetal deaths.

There was one patient with preeclampsia among the 13 cases; her serum screen was negative. One infant had a birth weight less than the tenth percentile for gestational age, weighing $2440 \mathrm{~g}$ at 37 weeks gestational age; her serum screen was also negative.

\section{DISCUSSION}

This study reveals that women with sickle cell disease have an increased chance of having a positive serum screen for Down syndrome, with a risk approaching $40 \%$. Only the hCG analyte was significantly greater in cases than controls; however, inhibin had a trend toward significance.

Although our study design does not substantiate conclusions about the mechanism of these findings, the analyte differences found are consistent with what is seen in pregnancies with placentation abnormalities. The underlying placentation problem with sickle cell disease is thought to be from placental hypoxia rather than a decidual or spiral arteriole invasion abnormality. ${ }^{4}$ Both inhibin A and hCG are produced in the syncytiotrophoblast, thus these two markers would be potentially affected by a similar insult in placental development. Hypoxia can induce cytotrophoblast proliferation and increased hCG production. Increased cytotrophoblast proliferation has also been noted in placentas of women with sickle disease. ${ }^{5}$ Thus, sickle cell disease could plausibly be associated with elevations in these two serum analytes.

If trophoblast proliferation is the underlying reason for increased hCG and inhibin A, we might not have expected to see changes in serum AFP or estriol. AFP is solely produced by the fetus and may be elevated when the placenta is more permeable, increasing diffusion into the maternal blood stream. The fetal precursor of estriol, 16-OH estrone sulfate, is converted by steroid sulfatase in the syncytiotrophoblast to estriol. Estriol is a dual fetal and placental product that tends to be low in pregnancies with disorders related to placentation abnormalities. ${ }^{3}$

There are a couple of major limitations to this study in addition to it being a retrospective cohort study with a small

Table 3 Comparison of serum analyte values

\begin{tabular}{lcccc} 
& Cases $(n=13)$ & Controls $(n=91)$ & RR (95\% CI) & $P$-value \\
\hline Positive screen & $5(38.5 \%)$ & $8(7.7 \%)$ & $7.50(1.60-35.8)$ & 0.0014 \\
Maternal age & $24.9 \pm 5.29$ & $26.8 \pm 5.29$ & 0.313 \\
\hline AFP MoM (mean \pm SD) & $1.00 \pm 0.44$ & $1.09 \pm 0.39$ & 0.47 \\
hCG MoM (mean \pm SD) & $2.00 \pm 1.65$ & $1.30 \pm 0.76$ & 0.017 \\
\hline Estriol MoM (mean \pm SD) & $0.94 \pm 0.53$ & $0.95 \pm 0.30$ & 0.914 \\
\hline Inhibin A MoM (mean \pm SD) & $1.70 \pm 1.53$ & $1.08 \pm 0.51$ & 0.114 \\
\hline
\end{tabular}

RR, relative risk; Cl, confidence interval; AFP, alpha-fetoprotein; hCG, human chorionic gonadotrophin; SD, standard deviation. 
number of subjects. We feel we had good ascertainment of all women with sickle cell disease seen at our institution. However, the control group was identified from both the high risk and prenatal database and does not reflect the general obstetrical population, thus there likely was enrichment for African American women with a positive Down syndrome screen. This would explain why $7.7 \%$ of the control population had a positive screen as opposed to the expected $2 \%$ for the general population based on the average age. Despite this higher than expected number of positive screens in the control population, we found a significant difference.

A second limitation is that serum screening in California changed twice during the course of the study, going from triple marker at the beginning of the time period, to quad screening in 2007, and then sequential screening in 2009. This then limited the number of cases (and controls) with inhibin A measurements. We found a trend toward significant differences with inhibin A, which may have been significant had this been measured throughout the entire study time frame. The limited data on first-trimester cases prevents finding an association with false positive first-trimester screens.
This study should be used as an aid in counseling women with sickle cell disease who have a positive serum screen for Down syndrome. This does not negate the possibility of the fetus actually having Down syndrome, but it can help give an explanation about why the test was positive. Further study is needed to determine if the risk for placenta-related disorders can be further defined using these maternal serum screening values, as pregnancies in women with sickle cell disease are well known to be at increased risk for preeclampsia ${ }^{6}$ and fetal growth restriction. ${ }^{7}$ This study should also provide guidance when counseling women about the interpretation of their prenatal screening results.

\section{WHAT'S ALREADY KNOWN ABOUT THIS TOPIC?}

- There are no previously published studies on the effect of sickle cell anemia on accuracy of maternal serum genetic screening.

\section{WHAT DOES THIS STUDY ADD?}

- Maternal serum second-trimester genetic screening has a high false positive rate in women with sickle cell anemia.

\section{REFERENCES}

1. Wald NJ, Rodeck C, Hackshaw AK, et al. First and second trimester antenatal screening for Down's syndrome: the results of the Serum, Urine and Ultrasound Screening Study (SURUSS). J Med Screen 2003;10(2):56-104.

2. Malone FD, Canick JA, Ball RH, et al. First-trimester or second-trimester screening, or both, for Down's syndrome. N Engl J Med 2005;353(19):2001-11.

3. Dugoff L. First- and second-trimester maternal serum markers for aneuploidy and adverse obstetric outcomes. Obstet Gynecol 2010;115(5):1052-61.

4. Trampont P, Roudier M, Andrea A, et al. The placental-umbilical unit in sickle cell disease pregnancy: a model for studying in vivo functional adjustments to hypoxia in humans. Hum Pathol 2004;35(11):1353-9.

5. Rathod KB, Jaiswal KN, Shrivastave AC, Shrikhande AV. Study of placenta in sickle cell disorders. Indian J Pathol Microbiol 2007;50 (4):698-701.

6. Villers MS, Jamison MG, De Castro LM, James AH. Morbidity associated with sickle cell disease in pregnancy. Am J Obstet Gynecol 2008;199 (2):125.e1-5.

7. Sun PM, Wilburn W, Raynor BD, Jamieson D. Sickle cell disease in pregnancy: twenty years of experience at Grady Memorial Hospital, Atlanta, Georgia. Am J Obstet Gynecol 2001;184(6):1127-30. 